\title{
EXTREME VALUES OF THE RIEMANN ZETA FUNCTION ON THE 1-LINE
}

\author{
CHRISTOPH AISTLEITNER, KAMALAKSHYA MAHATAB, AND MARC MUNSCH
}

\begin{abstract}
We prove that there are arbitrarily large values of $t$ such that $|\zeta(1+i t)| \geq e^{\gamma}\left(\log _{2} t+\log _{3} t\right)+\mathcal{O}(1)$. This essentially matches the prediction for the optimal lower bound in a conjecture of Granville and Soundararajan. Our proof uses a new variant of the "long resonator" method. While earlier implementations of this method crucially relied on a "sparsification" technique to control the mean-square of the resonator function, in the present paper we exploit certain self-similarity properties of a specially designed resonator function.
\end{abstract}

\section{INTRODUCTION}

Improving earlier work of Littlewood, in 1972 Levinson [7] proved that

$$
|\zeta(1+i t)| \geq e^{\gamma} \log _{2} t+\mathcal{O}(1)
$$

for arbitrarily large $t 1$ This was further improved by Granville and Soundararajan [5], who in 2006 established the lower bound

$$
\max _{t \in[0, T]}|\zeta(1+i t)| \geq e^{\gamma}\left(\log _{2} T+\log _{3} T-\log _{4} T+\mathcal{O}(1)\right)
$$

for arbitrarily large $t$. Their result also gives bounds for the measure of those $t \in[0, T]$ for which $|\zeta(1+i t)|$ is of this size. Also in [5], Granville and Soundararajan state the conjecture that actually

$$
\max _{t \in[T, 2 T]}|\zeta(1+i t)|=e^{\gamma}\left(\log _{2} T+\log _{3} T+C_{1}+o(1)\right),
$$

and even give a conjectural value of the constant $C_{1}$.

The proofs of Levinson and of Granville and Soundararajan rely on estimates for high moments of the zeta function and on Diophantine approximation arguments, respectively. Using a different method, the so-called resonance method, Hilberdink [6] re-established (11). This method can be traced back to a paper of Voronin [11, but it was developed independently and significantly refined by Hilberdink and by Soundararajan [9] about 10

\footnotetext{
The first and third author are supported by the Austrian Science Fund (FWF), project Y-901. This paper was written while the second author was a visitor at the TU Graz for one month; this visit was also supported by FWF project Y-901. Furthermore, this work was carried out during the tenure of an ERCIM "Alain Bensoussan" Fellowship of the second author.

${ }^{1}$ Throughout this paper, we write $\zeta(s)$ for the Riemann zeta function and $\log _{j}$ for the $j$-th iterated logarithm.
} 
years ago. Roughly speaking, the functional principle of this method is to find a function $R(t)$ such that

$$
I_{1}:=\int_{0}^{T} \zeta(\sigma+i t)|R(t)|^{2} d t
$$

is "large", whereas

$$
I_{2}:=\int_{0}^{T}|R(t)|^{2} d t
$$

is "small". Then the quotient $\left|I_{1}\right| / I_{2}$ is a lower bound for the maximal value of $|\zeta(\sigma+i t)|$ in the range $t \in[0, T]$. The "resonator" $R(t)$ is chosen as a Dirichlet polynomial, and experience shows that it is often suitable to choose a function $R$ with multiplicative coefficients which can be written as a finite Euler product. This method can be implemented relatively easily if the length of $R$ is bounded by a small power of $T$, since then $I_{2}$ is the squareintegral of a sum of essentially orthogonal terms. However, it is desirable to be able to control significantly longer resonator functions as well, and in [1] a method was developed which allows the implementation of "long" resonators of length roughly $T^{\log _{2} T}$. For $\sigma \in(1 / 2,1)$ this method allowed to recapture Montgomery's [8] lower bounds for extreme values of $|\zeta(\sigma+i t)|$ by means of the resonance method, while on the critical line Bondarenko and Seip [3] used a "long resonator" to obtain lower bounds for extreme values of $|\zeta(1 / 2+i t)|$ which even surpassed those established before by different methods 2 In the present paper we will adapt the "long resonator" argument to the case $\sigma=1$, and prove the following theorem.

Theorem 1. There is a constant $C$ such that

$$
\max _{t \in[\sqrt{T}, T]}|\zeta(1+i t)| \geq e^{\gamma}\left(\log _{2} T+\log _{3} T-C\right)
$$

for all sufficiently large $T$.

Note that our theorem is in accordance with the conjecture of Granville and Soundararajan in equation (2). However, the conjecture is much stronger than the theorem. First of all, our theorem only gives a lower bound with error $\mathcal{O}(1)$, while the conjecture gives an asymptotic equality with error $o(1)$. Thus a proof of the full conjecture would also require a vast improvement of the upper bound $|\zeta(1+i t)| \ll(\log t)^{2 / 3}$ of Vinogradov. Additionally, in the conjecture the range for $t$ is $[T, 2 T]$, while our theorem requires the larger range $[\sqrt{T}, T]$. The requirement for such a longer range is typical for applications of the "long resonator", and appears in [1] and [3] as well. The range $[\sqrt{T}, T]$ in our theorem could be reduced to $\left[T^{\theta}, T\right]$ for $\theta<1$, at the expense of replacing $C$ by some other constant $C(\theta)$.

Before turning to the proof of Theorem 1, we comment on the difficulties when extending the "long resonator" method to the case $\sigma=1$. Two key ingredients of the resonance method for a "long resonator" are positivity and sparsity. "Positivity" means the introduction of an additional function

\footnotetext{
${ }^{2}$ From the work of Bondarenko and Seip it is also visible why the "long resonator" does not give any essential improvement of Montgomery's results in the case $\sigma \in(1 / 2,1)$, other than better values for the involved constants. See 3, 4,
} 
having non-negative Fourier transform in $I_{1}$, which ensures that $I_{1}$ is a sum of non-negative terms, while "sparsity" means that nearby frequencies in the resonator function are merged in such a way that one obtains a function having a "quasi-orthogonality" property which allows to control $I_{2}$. For details see [1, 3] and the very recent paper [4]. The "long resonator" has only been implemented for $\sigma$ in the range $[1 / 2,1)$ so far, since the extension of the method to $\sigma=1$ meets serious technical difficulties. The main problem is that the "sparsification" of the resonator cannot be carried out to such an extent as to obtain a truly orthogonal sum, but one rather ends up with a function whose square-integral can be estimated only up to multiplicative errors of logarithmic order; if one tried to continue thinning out the resonator function to obtain precise control of $I_{2}$, then from some point on this would cause a significant loss in $I_{1}$ instead. Note that these errors do not play a role in the case $\sigma \in[1 / 2,1)$, where they are negligible in comparison to the main terms, whereas in the case $\sigma=1$ we want to obtain a very precise result where only multiplicative errors of order $\left(1+1 / \log _{2} T\right)$ are allowed.

Thus for the case $\sigma=1$ it is necessary to devise a novel variant of the "long resonator" technique, which will be done in the present paper. The method is genuinely different from those used in 1, 3, where the "sparsification" of the resonator function played a key role. In the present paper we will avoid this sparsity requirement, and actually we make no attempt at all to control the size of $I_{2}$. Instead we use a self-similarity property of the resonator function, which is due to its construction in a completely multiplicative way.

\section{Proof of Theorem 1}

We will use the following approximation formula for the zeta function, which appears in the first lines of the proof of Theorem 2 in [5].

Lemma 1. Define $\zeta(s ; Y):=\prod_{p \leq Y}\left(1-p^{-s}\right)^{-1}$. Let $T$ be large, and set $Y=\exp \left((\log T)^{10}\right)$. Then for $T^{1 / 10} \leq|t| \leq T$ we have

$$
\zeta(1+i t)=\zeta(1+i t ; Y)\left(1+\mathcal{O}\left(\frac{1}{\log T}\right)\right) .
$$

Instead of this approximation of $\zeta$ by an Euler product we could also use the classical approximation by a Dirichlet polynomial ([10, Theorem 4.11]), which was used in 1, 3, 6]. However, the approximation by an Euler product is more convenient for us, since the resonator will also be defined as an Euler product.

Assume that $T$ is "large", and set $Y=\exp \left((\log T)^{10}\right)$. By Lemma 1 it suffices to prove Theorem 1 for $\zeta(1+i t ; Y)$ instead of $\zeta(1+i t)$. Set $X=(\log T)\left(\log _{2} T\right) / 6$, and for primes $p \leq X$ set

$$
q_{p}=\left(1-\frac{p}{X}\right) \text {. }
$$

This choice of "weights" is inspired by those used in the proof of Theorem 2.3 (case $\alpha=1$ ) in [6]. We set $q_{1}=1$ and $q_{p}=0$ for $p>X$, and extend our 
definition in a completely multiplicative way such that we obtain weights $q_{n}$ for all $n \geq 1$. Now we define

$$
R(t)=\prod_{p \leq X}\left(1-q_{p} p^{i t}\right)^{-1}
$$

Then

$$
\begin{aligned}
\log (|R(t)|) & \leq \sum_{p \leq X}(\log X-\log p) \\
& =\pi(X) \log X-\vartheta(X),
\end{aligned}
$$

where $\pi$ is the prime-counting function and $\vartheta$ is the first Chebyshev function. It is well-known that by partial summation one has

$$
\pi(X) \log X-\vartheta(X)=\int_{2}^{X} \frac{\pi(t)}{t} d t=(1+o(1)) \frac{X}{\log X},
$$

and thus we have

$$
|R(t)|^{2} \leq T^{1 / 3+o(1)}
$$

by our choice of $X$.

We can write $R(t)$ as a Dirichlet series in the form

$$
R(t)=\sum_{n=1}^{\infty} q_{n} n^{i t}
$$

and accordingly

$$
|R(t)|^{2}=\sum_{m, n=1}^{\infty} q_{m} q_{n}\left(\frac{m}{n}\right)^{i t} .
$$

Note that all the weights $q_{n}, n \geq 1$, are non-negative reals. Set $\Phi(t)=e^{-t^{2}}$. By our choice of $Y$ we have

$$
|\zeta(1+i t ; Y)| \ll \log Y \ll(\log T)^{10} .
$$

Thus, using (4), we obtain

$$
\left.\left|\int_{|t| \geq T} \zeta(1+i t ; Y)\right| R(t)\right|^{2} \Phi\left(\frac{\log T}{T} t\right) d t \mid \ll 1
$$

and

$$
\left.\left|\int_{|t| \leq \sqrt{T}} \zeta(1+i t ; Y)\right| R(t)\right|^{2} \Phi\left(\frac{\log T}{T} t\right) d t \mid \ll T^{5 / 6+o(1)} .
$$

Using (5) we can write

$$
\begin{aligned}
I_{2} & :=\int_{-\infty}^{\infty}|R(t)|^{2} \Phi\left(\frac{\log T}{T} t\right) d t \\
& =\sum_{m, n=1}^{\infty} \int_{-\infty}^{\infty} q_{m} q_{n}\left(\frac{m}{n}\right)^{i t} \Phi\left(\frac{\log T}{T} t\right) d t .
\end{aligned}
$$

We have the lower bound

$$
\int_{\sqrt{T}}^{T}|R(t)|^{2} \Phi\left(\frac{\log T}{T} t\right) d t \gg T^{1+o(1)}
$$


which follows from $q_{1}=1$ and the positivity of the Fourier transform of $\Phi$, together with estimates similar to (6) and (7) to restrict to the desired range. Again using the fact that $\Phi$ has a positive Fourier transform we have

$$
\begin{aligned}
& \int_{-\infty}^{\infty} \zeta(1+i t ; Y)|R(t)|^{2} \Phi\left(\frac{\log T}{T} t\right) d t \\
\geq & \int_{-\infty}^{\infty} \zeta(1+i t ; X)|R(t)|^{2} \Phi\left(\frac{\log T}{T} t\right) d t .
\end{aligned}
$$

Here we reduced the range of primes in $\zeta(1+i t ; \cdot)$ from $Y$ to $X$, so that the same primes appear in the definitions of $\zeta(1+i t ; \cdot)$ and $R$, respectively. Writing

$$
\zeta(1+i t ; X)=\sum_{k=1}^{\infty} a_{k} k^{-i t}
$$

for appropriate coefficients $\left(a_{k}\right)_{k \geq 1}$, where $a_{k} \in\{0,1 / k\}, k \geq 1$, we have

$$
\begin{aligned}
I_{1} & :=\int_{-\infty}^{\infty} \zeta(1+i t ; X)|R(t)|^{2} \Phi\left(\frac{\log T}{T} t\right) d t \\
& =\sum_{k=1}^{\infty} a_{k} \sum_{m, n=1}^{\infty} \int_{-\infty}^{\infty} k^{-i t} q_{m} q_{n}\left(\frac{m}{n}\right)^{i t} \Phi\left(\frac{\log T}{T} t\right) d t .
\end{aligned}
$$

Note that we can freely interchange the order of summations and integration, since everything is absolutely convergent. Assume $k$ to be fixed. Then, again using the fact that $\Phi$ has a positive Fourier transform, we have

$$
\begin{aligned}
& \sum_{m, n=1}^{\infty} \int_{-\infty}^{\infty} k^{-i t} q_{m} q_{n}\left(\frac{m}{n}\right)^{i t} \Phi\left(\frac{\log T}{T} t\right) d t \\
\geq & \sum_{n=1}^{\infty} \sum_{\substack{1 \leq m<\infty \\
k \mid m}} \int_{-\infty}^{\infty} k^{-i t} q_{m} q_{n}\left(\frac{m}{n}\right)^{i t} \Phi\left(\frac{\log T}{T} t\right) d t \\
= & \sum_{n=1}^{\infty} \sum_{r=1}^{\infty} \int_{-\infty}^{\infty} k^{-i t} \underbrace{q_{r k}}_{=q_{r} q_{k}} q_{n}\left(\frac{r k}{n}\right)^{i t} \Phi\left(\frac{\log T}{T} t\right) d t \\
= & q_{k} \underbrace{\sum_{n=1}^{\infty} \sum_{r=1}^{\infty} \int_{-\infty}^{\infty} q_{r} q_{n}\left(\frac{r}{n}\right)^{i t} \Phi\left(\frac{\log T}{T} t\right) d t .}_{=I_{2}}
\end{aligned}
$$

Thus we have

$$
\begin{aligned}
\frac{I_{1}}{I_{2}} & \geq \sum_{k=1}^{\infty} a_{k} q_{k} \\
& =\prod_{p \leq X}\left(1-q_{p} p^{-1}\right)^{-1} \\
& =\left(\prod_{p \leq X}\left(1-p^{-1}\right)^{-1}\right)\left(\prod_{p \leq X} \frac{p-1}{p-q_{p}}\right) .
\end{aligned}
$$


For the first product we have

$$
\prod_{p \leq X}\left(1-p^{-1}\right)^{-1}=e^{\gamma} \log X+\mathcal{O}(1)=e^{\gamma}\left(\log _{2} T+\log _{3} T\right)+\mathcal{O}(1)
$$

by Mertens' theorem. For the second product we have

$$
\begin{aligned}
-\log \left(\prod_{p \leq X} \frac{p-1}{p-q_{p}}\right) & =-\sum_{p \leq X} \log \left(1-\frac{p}{p+(p-1) X}\right) \\
& \ll \sum_{p \leq X} \frac{1}{X} \\
& \ll \frac{1}{\log X} .
\end{aligned}
$$

Thus together with (10) and (11) we have

$$
\frac{I_{1}}{I_{2}} \geq e^{\gamma}\left(\log _{2} T+\log _{3} T\right)+\mathcal{O}(1) \text {. }
$$

From (6)-(9) we deduce that also

$$
\frac{\left.\left|\int_{\sqrt{T}}^{T} \zeta(1+i t ; Y)\right| R(t)\right|^{2} \Phi\left(\frac{\log T}{T} t\right) d t \mid}{\int_{\sqrt{T}}^{T}|R(t)|^{2} \Phi\left(\frac{\log T}{T} t\right) d t} \geq e^{\gamma}\left(\log _{2} T+\log _{3} T\right)+\mathcal{O}(1) .
$$

Thus we have

$$
\max _{t \in[\sqrt{T}, T]}|\zeta(1+i t ; Y)| \geq e^{\gamma}\left(\log _{2} T+\log _{3} T\right)+\mathcal{O}(1) .
$$

As noted at the beginning of the proof, by Lemma 1 this estimate remains valid if we replace $\zeta(1+i t ; Y)$ by $\zeta(1+i t)$. This proves Theorem 1 .

In conclusion, we add some remarks on the method used in the present paper. As noted in the introduction, earlier implementations of the resonance method relied on a combination of "positivity" and "sparsity" properties. In the present paper we show that it is possible to leave out the sparsity requirement, at least in one particular instance. One could also obtain the results from [1] for the case $\sigma \in(1 / 2,1)$ using the method from the present paper without problems. However, it does not seem that the results from [3] for the case $\sigma=1 / 2$ could also be obtained using our argument, since the resonator function there necessarily has a more complicated structure (which is not of a simple Euler product form), while our argument relies on the fact that one can use a resonator which has completely multiplicative coefficients (which is essential for the argument, since any other coefficients would destroy the required self-similarity property).

We want to emphasize that the only real restriction on the size of the resonator in our argument is (4), which gives an upper bound for $|R(t)|$. This is different from earlier versions of the "long resonator" argument, where bounds on the cardinality of the support of $R(t)$ (that is, on the number of non-zero coefficients in the Dirichlet series representation) are required. 
Another remark is that while the sparsity requirement may be unnecessary (at least in some cases), the positivity requirement still plays a crucial role in our argument. This prevents a possible generalization of the method to the case of functions whose Dirichlet series representation does not only contain non-negative real numbers as coefficients. This topic is also discussed in 2] in some detail. In particular, we have not been able to obtain a result for extreme values of $1 / \zeta$ beyond those mentioned in [5].

\section{REFERENCES}

[1] C. Aistleitner. Lower bounds for the maximum of the Riemann zeta function along vertical lines. Math. Ann., 365(1-2):473-496, 2016.

[2] C. Aistleitner and E. Pańkowski. Large values of L-functions from the Selberg class. J. Math. Anal. Appl., 446(1):345-364, 2017.

[3] A. Bondarenko and K. Seip. Large greatest common divisor sums and extreme values of the Riemann zeta function. Duke Math. J., 166(9):1685-1701, 2017.

[4] A. Bondarenko and K. Seip. Note on the resonance method for the Riemann zeta function. To appear in: Tribute to Victor Havin. 50 years with Hardy spaces, in the series "Operator Theory: Advances and Applications", Birkhäuser Verlag. Available at https://arxiv.org/abs/1701.04978.

[5] A. Granville and K. Soundararajan. Extreme values of $|\zeta(1+i t)|$. In The Riemann zeta function and related themes: papers in honour of Professor K. Ramachandra, volume 2 of Ramanujan Math. Soc. Lect. Notes Ser., pages 65-80. Ramanujan Math. Soc., Mysore, 2006.

[6] T. Hilberdink. An arithmetical mapping and applications to $\Omega$-results for the Riemann zeta function. Acta Arith., 139(4):341-367, 2009.

[7] N. Levinson. $\Omega$-theorems for the Riemann zeta-function. Acta Arith., 20:317-330, 1972.

[8] H. L. Montgomery. Extreme values of the Riemann zeta function. Comment. Math. Helv., 52(4):511-518, 1977.

[9] K. Soundararajan. Extreme values of zeta and L-functions. Math. Ann., 342(2):467486, 2008.

[10] E. C. Titchmarsh. The theory of the Riemann zeta-function. The Clarendon Press, Oxford University Press, New York, second edition, 1986. Edited and with a preface by D. R. Heath-Brown.

[11] S. M. Voronin. Lower bounds in Riemann zeta-function theory. Izv. Akad. Nauk SSSR Ser. Mat., 52(4):882-892, 896, 1988.

Christoph Aistleitner, Institute of Analysis and Number Theory, TU Graz, Austria

E-mail address: aistleitner@math.tugraz.at

Kamalakshya Mahatab, Department of Mathematical Sciences, NTNU TrondHEIM, NORWAY

E-mail address: accessing.infinity@gmail.com

Marc Munsch, Institute of Analysis and Number Theory, TU Graz, Austria

E-mail address: munsch@math.tugraz.at 